\title{
Stable and Reversible Functionalization and Super-Resolution Micros- copy of Live Cell Membranes
}

\author{
Ahad Hossain, a Joydeb Majumder, a Cheng Bi, b Fang Huang, b,c,d,e and Gaurav Chopra a,c,d,e,f* \\ a. Department of Chemistry, Purdue University, 560 Oval Drive, West Lafayette, IN 47907, USA \\ b. Purdue University, Weldon School of Biomedical Engineering, 206 S. Martin Jischke Drive, West Lafayette, IN 47907-
} 2032

c. Purdue Center for Cancer Research, Purdue University, 201 S University St, West Lafayette, IN 47906, USA

d. Purdue Institute for Integrative Neuroscience, Purdue University, 207 South Martin Jischke Drive, West Lafayette, IN 47907, USA

\begin{abstract}
e. Purdue Institute for Inflammation, Immunology and Infectious Disease, Purdue University, 610 Purdue Mall, West Lafayette, IN 47907, USA.
\end{abstract}

f. Purdue Institute for Drug Discovery, Purdue University, 720 Clinic Drive, West Lafayette, IN 47907, USA†

KEYWORDS Cell surface conjugation, live-cell imaging

\begin{abstract}
Live cell surface functionalization has been increasingly explored in recent years because of its emerging biomedical and therapeutic applications. Known methods to functionalize live-cell surface includes time-consuming metabolic docking of non-natural functional groups on cell surfaces that are hard to use. Here, we report an easy and direct functionalization method for cell surface and cell-cell conjugation utilizing native phosphate groups on cell membranes. We used three-dimensional single-molecule localization microscopy to confirm that the chemical conjugate binds preferentially on the cell surface. We show an application of this method for live-cell imaging and enhancement of cell-cell interaction leading to increased T cell proliferation. Our simple and reversible cell-conjugation strategy would be widely useful to explore and optimize cell-cell contact-based proliferation for any cell-based therapeutic applications.
\end{abstract}

\section{Introduction}

Surface modification of live cells has many biological applications including imaging, control of cell-surface interactions, tracking, and sensing biological environments in vitro and in vivo. ${ }^{1,2}$ Recently, cell surface functionalization has received significant attention from researchers and clinicians perhaps due to several biomedical applications. ${ }^{3,4}$ Several methods have been developed to functionalize the cell surface with cargo vehicles and therapeutic agents. Most popular conjugation methods include hydrophobic anchoring,5-14 chemoselective conjugation, ${ }^{15-22,23,24,25}$ PEGylation ${ }^{26}$ etc. Live cell surface modification is done to date show limited-time stability and may affect their desired function. For example, the overall stability for PEG-lipids conjugation on the cell surface was no longer than 1-2 days before all the PEG molecules dissociated from the cell surface. ${ }^{27}$ Furthermore, incorporating a hydrophobic chain in the polymer scaffold results in uptake by the cells affecting normal cell function. On the other hand, metal-free covalent conjugation of live cells by chemoselective reactions on transmembrane proteins provides slightly longer stability of the surface conjugation. ${ }^{28-30}$ but results in toxic effects and disruption of normal cellular function 31 due to non-specific binding of the cargo molecules with other amine groups on membrane proteins. Finally, there are also
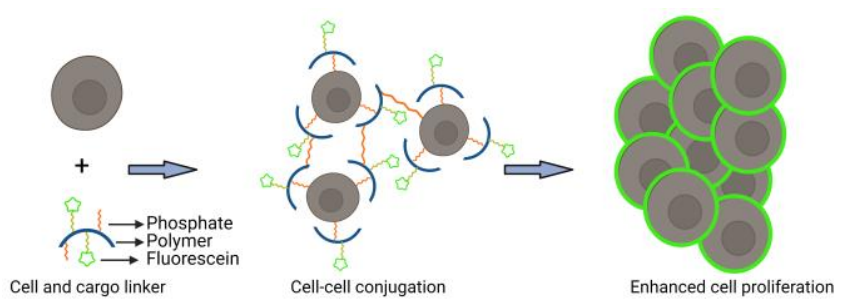

Figure 1. Schematic representation showing cell-cell conjugation through cargo linker to enhance cell-cell interactions leading to enhanced cell proliferation.

limitations in the development of nanoparticles-

based cell-conjugates for in vivo cell therapy. For example, nanoparticles cause toxic side effects after entrapment in the reticuloendothelial system of the liver and spleen ${ }^{32}$ and the bio-compatibility of synthetic nanoparticles made from 
inorganic materials raises a major concern for translation. ${ }^{33}$ Cell-based therapeutics have received significant attention in modern medicine because of their ability to treat intractable diseases such as cancer. ${ }^{34,35}$ Therapeutic cells, such as T cells or Chimeric Antigen Receptor Tcells (CAR-Ts) are promising for the treatment of diseases that currently are not curable by traditional small molecule drugs or biologics. ${ }^{36,37}$ However, one of the greatest challenges associated with these "living" drugs is their manufacturing. If more efficient time-economic biomanufacturing technologies can be developed for large-scale cellular therapeutic production, more patients could be treated with these lifesaving therapeutics. ${ }^{38}$ Therapeutic cells, such as tumor infiltrated lymphocytes, ${ }^{39}$ and CAR-Ts ${ }^{40}$ have been used as immunotherapeutic "drugs." Biomanufacturing of these cells is a labor-intensive process and any enhancement to reduce the ex vivo expansion time is desirable for large-scale cost-effective production of therapeutic cells. We hypothesized that if a macromolecular or polymer linker is designed with dual bond-forming moieties such as a cationic moiety for non-covalent bonding and phosphate functionality for covalent bonding, one side of this linker can conjugate the surface of one cell while the other side of that same linker can conjugate the surface of another cell. Such a linker would increase cellcell interactions enhancing the proliferation of cells - leading to an efficient and effective method for enhancing cell biomanufacturing (schematically shown in Figure 1).

Recently, several cell-surface conjugation methods have been developed to manipulate cellular interactions for exploiting various cell-based applications. ${ }^{41,42}$ Still, live-cell surface modification remains challenging because of a lack of chemically reactive functional groups on many cell surfaces and the dynamic structure of the plasma membrane. ${ }^{2}$ Poor stability of the conjugated linker on a cell surface is also another problem for long-term application. ${ }^{43}$ Other methods to install non-natural functional groups on cell surfaces via metabolic docking takes several days to express those functional groups. ${ }^{44,45,46,47,48,49}$ Thus, there is a need to develop a facile and general method for live cell surface functionalization without altering the function of the cells. Here, we provide a general cell surface conjugation strategy using the native phosphate groups on the cell membrane. We have decorated one side of the cargo molecule with a cationic functionality to facilitate non-covalent interaction with the negatively charged cell surface and the other side of the cargo molecule with a phosphoric acidcontaining ligand such as adenosine diphosphate (ADP) to facilitate phospho-ester covalent bonds under the physiological condition with the cell surface phospholipid phosphate functionalities. Our dual chemical conjugation approach provides longer stability of the cell surface conjugation and shows no effect on viability due to the formation of the natural phospho-ester bond instead of unnatural chemical reactions.

\section{RESULTS AND DISCUSSION}

To demonstrate the effect of small-molecule fluorophore conjugates on cells, we first synthesized an ADPfluorescein conjugate and treated Jurkat T cells. Like fluo- rescein treatment, ADP-fluorescein conjugate was internalized by T cells (Figure S1). This led to the design of a cargo molecule similar to a polymer/macromolecule carrier. We designed and synthesized a cargo backbone with only a side-chain cationic group (namely non-covalent cargo) while another cargo backbone with both side-chain cationic and phosphate groups (namely covalent cargo, Figure S2a-b, Figure 2a-b). To test our hypothesis, we also synthesized phospholipid linked non-covalent and covalent cargos (Figure S2c and Figure S3) and characterized by

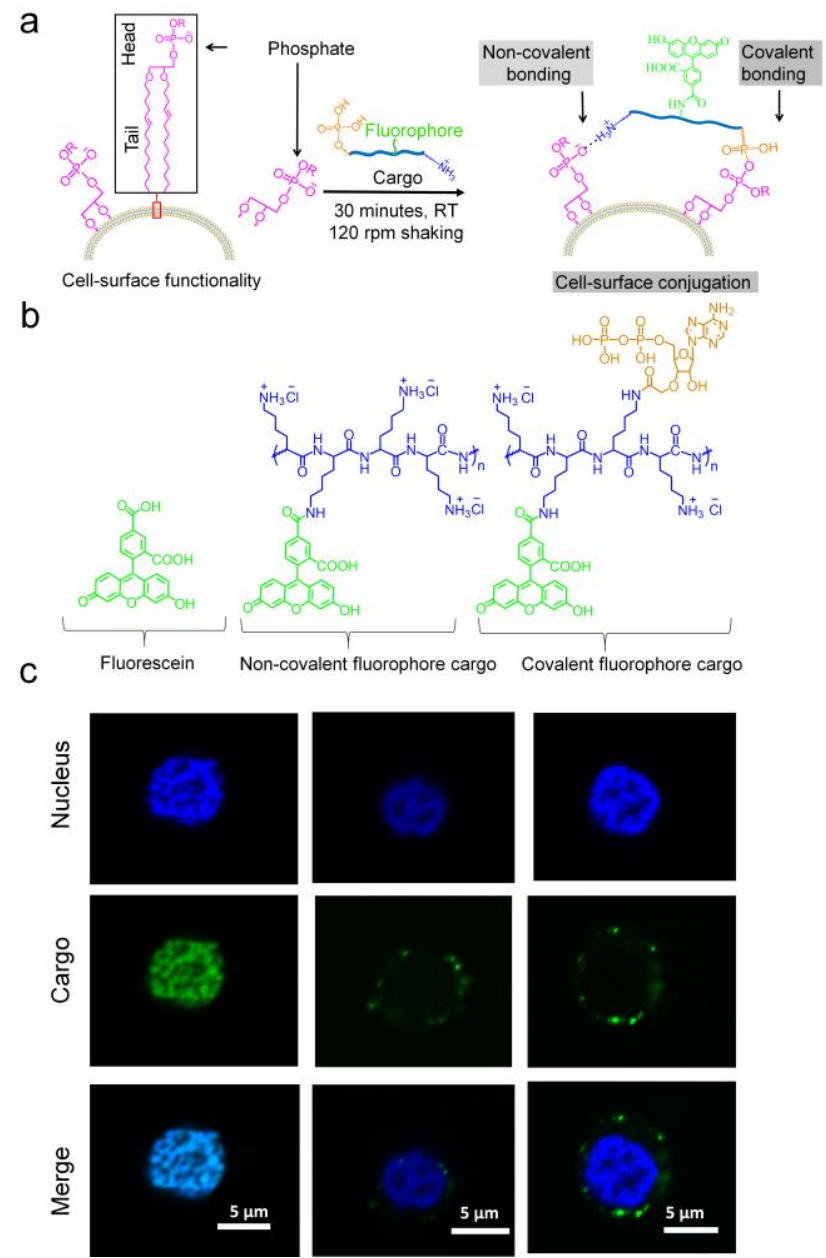

Figure 2. Chemical structures for live-cell surface conjugation. (a) Cell surface functionality with negatively charged phosphate. Covalent and non-covalent conjugation of the fluorophore-linked cargos on the cell surface. (b) Structures of the fluorescein, non-covalent and covalent cargos tagged with fluorescein. (c) Confocal images of the Jurkat $\mathrm{T}$ cells after treatment with fluorescein and fluorescein-tagged cargo molecules and followed by Hoechst 33342 nuclear staining. Scale bars represent $5 \mu \mathrm{m}$.

\section{NMR and FT-IR spectroscopy (Figure S18-26).}

For phospholipid fatty acid, $\mathrm{P}=0$ stretching band appeared at $1170 \mathrm{~cm}^{-1}$, and the $\mathrm{P}=0$ stretch was at $1156 \mathrm{~cm}^{-1}$ for non-covalent cargo (Figure S24). The decrease in $\mathrm{P}=0$ 
stretching band indicated its presence as anion and/or conjugated form such as a phospho-ammonium group. Similarly, for covalent phospholipid cargo, a larger decrease in $\mathrm{P}=0$ stretching band suggests a covalent bond in extended conjugated form as a tri-phosphoester bond. The decrease in $\mathrm{P}=0$ stretching band was observed at $972 \mathrm{~cm}^{-1}$ for covalent phospholipid, compared to $1103 \mathrm{~cm}^{-1}$ in ADP and $1075 \mathrm{~cm}^{-1}$ in the covalent cargo (Figure S25). After establishing bond formation between our synthetic cargos and the phospholipids, we hypothesized that our noncovalent and covalent cargo molecules will interact with the native cell-surface phospholipids, as outlined in Figure 2a. We conjugated a fluorophore molecule (fluorescein) with both non-covalent and covalent cargo molecules to locate the presence of the cargo molecules on cells (Figure S4-5). Next, we were interested to see whether these cargo molecules will conjugate on the membrane of Jurkat $\mathrm{T}$ cells. We identified both optimal molecular weight and concentration for these cargos (Figure S6-7) and used it with an optimal concentration of $0.1 \mathrm{mg} / \mathrm{mL}$ to treat the cells at RT for 30 minutes on an orbital shaker followed by stained with Hoechst 33342. Fluorescein was internalized by the cells as observed by the perfect overlap of blue fluorescence of Hoechst 33342 and green fluorescence of fluorescein in the nucleus of the Jurkat cells. When we used the fluorescein-conjugated cargo, the blue fluorescence of Hoechst was observed in the nucleus and green fluorescence of fluorescein was observed at the periphery of the cells (Figure 2c). The live-cell surface conjugation efficiency of the covalent cargo was better than the non-covalent cargo; suggesting that our hypothesis to develop a dualfunctional covalent cargo was rational for cell-surface conjugation. Next, using various length D-lysine linker backbone, we synthesized three covalent cargos 1 to 3 with molecular weights of 75,150 , and $300 \mathrm{kD}$ respectively (Figure S8a). We treated the cells with $0.1 \mathrm{mg} / \mathrm{mL}$ of the cargo 1-3 at RT for 30 minutes on an orbital shaker. Then, cells were washed, stained with Hoechst 33342, and imaged via fluorescence confocal microscopy (Figure S8b). The live-cell surface conjugation efficiency of the cargo 2 (150 kD) was better than the other cargos. Since keeping cells alive after cell surface conjugation is a requirement for future applications. We performed the viability test of the surface-modified Jurkat $\mathrm{T}$ cells for 3 days by cell-titer blue viability assay, which resulted in more than $80 \%$ viability of the surface-modified Jurkat T cells (Figure S8c) for all these cargos. To further validate the usability of our reagent for membrane imaging of fixed cells we also treated our dual covalent cargo with fixed Jurkat $\mathrm{T}$ cells and observed membrane imaging of cells (Figure S9). These results suggest that our stable and non-toxic conjugate reagent can be used to image both fixed and live cells. This was a useful application of our cargo 2 - since there are very limited and very expensive non-toxic staining reagents for live cell membrane imaging that do not show long-term stability. Furthermore, to verify the need for dual-cell conjugation, we synthesized a cargo by protecting its cationic ammonium side chains with BOC-groups. We observed no surface conjugation of the Jurkat T cells with the BOC-cargo indicating the plausible role of the cargo decoration chemistry for cell-surface bond formations (Figure S10).

A major objective of this work is to develop a cell-surface conjugation method with long-time stability under physiological conditions $\left(37^{\circ} \mathrm{C}\right.$ and $\mathrm{pH}$ 7.4) for several days. To our knowledge, the current stability of live cell conjugation, using artificial techniques that are hard to use for multiple cell types, is less than 72 hours. ${ }^{10}$ We incubated surface-modified Jurkat $\mathrm{T}$ cells at $37^{\circ} \mathrm{C}$ and $\mathrm{pH} 7.4$ for a period of 6 days by simply adding our cargo molecules to the culture. The images were recorded after 1,3 , and 6 days (Figure 3a and figure S10). Both the non-covalent and covalent cargo molecules show stability to the surface of the Jurkat T cells - as revealed by green fluorescence on the cell membrane after one day of conjugation but the covalent cargo molecule shows stability with more than $50 \%$ fluorescent remaining after six days in culture (Figure $3 \mathbf{b}$ ). It is well known that activated T-cells result in lower surface redox levels with a reduced number of

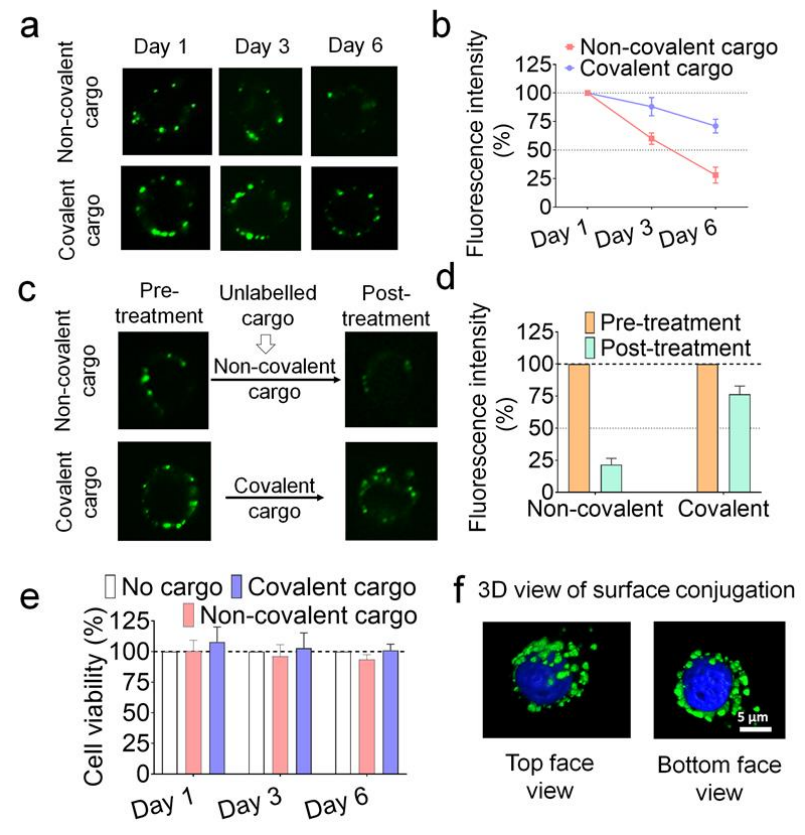

Figure 3. Stability and viability and of the surface-modified Jurkat T cells. (a) Zoomed in view of confocal laser microscopic images of the surface-modified Jurkat $\mathrm{T}$ cells conjugated with both the cargos after 1, 3, and 6 days. (b) Quantification of surface fluorescence intensity of cells in Figure 3a. (c) Stability of the surface conjugated cells in the presence of no fluorophore tag respective cargos. (d) Quantification of surface fluorescence intensity of cells in Figure 3c. (d) Quantification of surface fluorescence intensity of cells in Figure 3c. (e) Viability of Jurkat T cells after their surface conjugation with the cargos. (f) Volume view of surface conjugated Jurkat $\mathrm{T}$ cells with covalent cargo. Dual-conjugation of the covalent cargo with Jurkat $\mathrm{T}$ cell is cytocompatible and stable under physiological conditions $\left(37^{\circ} \mathrm{C}\right.$ and $\mathrm{pH}$ of 7.4$)$ over 6 days.

membrane thiol (-SH) groups. ${ }^{50,51}$ We verified the stability of our cargo with interleukin-2 (IL-2) activated Jurkat T cells (Figure S12) suggesting the possible use of conjugated immune cell therapy as future applications. This data 
suggests long-time stability of the surface conjugation for the dual covalent and non-covalent conjugation compared to only non-covalent cell conjugation. To investigate whether the phosphate bearing groups of the covalent cargo have stable bonding with the cell surface functionality in a competing environment, we conjugated Jurkat $\mathrm{T}$ cells with fluorophore tag covalent cargo for 30 minutes and then washed with PBS. Next, the cells were treated with a covalent cargo molecule with no fluorophore tag (competing for similar bonding with florescent cargo molecules) for 30 minutes in growth media at $120 \mathrm{rpm}$ at room temperature.

We also performed the same experiment with a noncovalent florescent cargo molecule in a competing presence of no fluorophore tagged non-covalent cargo $(150 \mathrm{kD}$ D-lysine chain polymer) for 30 minutes. The results indicate that the green fluorescence intensity on the surface of live cells conjugated with fluorophore-tag non-covalent cargo reduced significantly, compared to fluorophore-tag covalent cargo cell surface conjugation (Figure 3c-d and Figure S13). ). Our data indicate additional bonding interactions of the dual-conjugated covalent cargo, compared to the non-covalent cargo, with cell surface based on our cargo design strategies. Keeping cells alive after cell surface conjugation is a requirement for future applications. To see the cytotoxic effect of surface conjugation, we performed a viability test of the surface-modified Jurkat T cells for 6 days. We performed the cell-titer blue viability assay resulting in more than 95\% surface-modified Jurkat T cells viability after 6 days (Figure 3e). Confocal 3D images of surface conjugated Jurkat $\mathrm{T}$ cell with the stable and nontoxic covalent fluorophore cargo is shown in Figure 3f.

. To confirm, the "blobs" we were seeing in the confocal imaging are the cargo conjugate surrounding the cells, we performed super-resolution imaging (Figure 4). Here, we prepared a new cargo conjugated with Alexa Fluor 647 (hereafter, referred to as AF647) for three-dimensional single-molecule super-resolution microscopy using a biplane configuration. With the inferred axial position of the localized single molecules encoded in color, we observe the three-dimensional distribution of AF647 labeled cargo on the cell surface confirming our hypothesis that the polymer conjugate binds preferentially towards the cell surface membrane.

Next, we were interested to study the reversibility and reusability of the covalent cargo reagent for live-cell imaging. We conjugated a magnetic bead to our covalent cargo that was designed to form bonds with the cell membrane such that the bonds are stimulated by $\mathrm{pH}$. The magnetic beads were essential to isolate the cargo reagent, both from excess cargo that was not conjugated to cells at 0.1 $\mathrm{mg} / \mathrm{mL}$ concentration during initial conjugation (unconjugated magnetic cargo), as well as, after the cell-surface bond disruption and recovery of the cell conjugated cargo reagent by $\mathrm{pH}$ stimulation ( $\mathrm{pH}$ recovered magnetic cargo), as shown in the schematic representation in Figure S14. Covalent cargo conjugated Jurkat $\mathrm{T}$ cells were treated with PBS at pH 5.5 to disrupt surface conjugation, observed after 30 minutes of incubation, as evident from no green fluorescence on the cell surface. Next, the mixture of T cells and $\mathrm{pH}$-stimulated unconjugated magnetic cargo reagents were recovered using magnetic isolation from the supernatant.

The $\mathrm{pH}$ recovered magnetic cargo solution was adjusted to pH 7.0 and reused in combination with the unconjugated magnetic cargo to treat live Jurkat T cells. Surface conjugation of Jurkat $\mathrm{T}$ cells was observed for the combination of

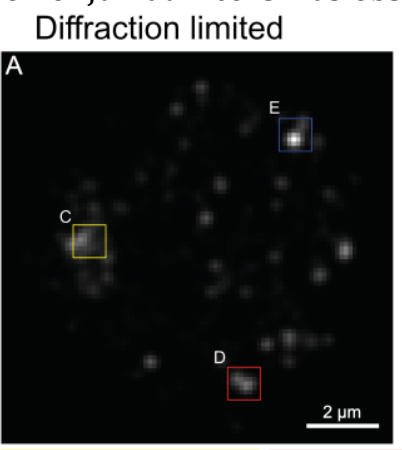
3D Super-resolution
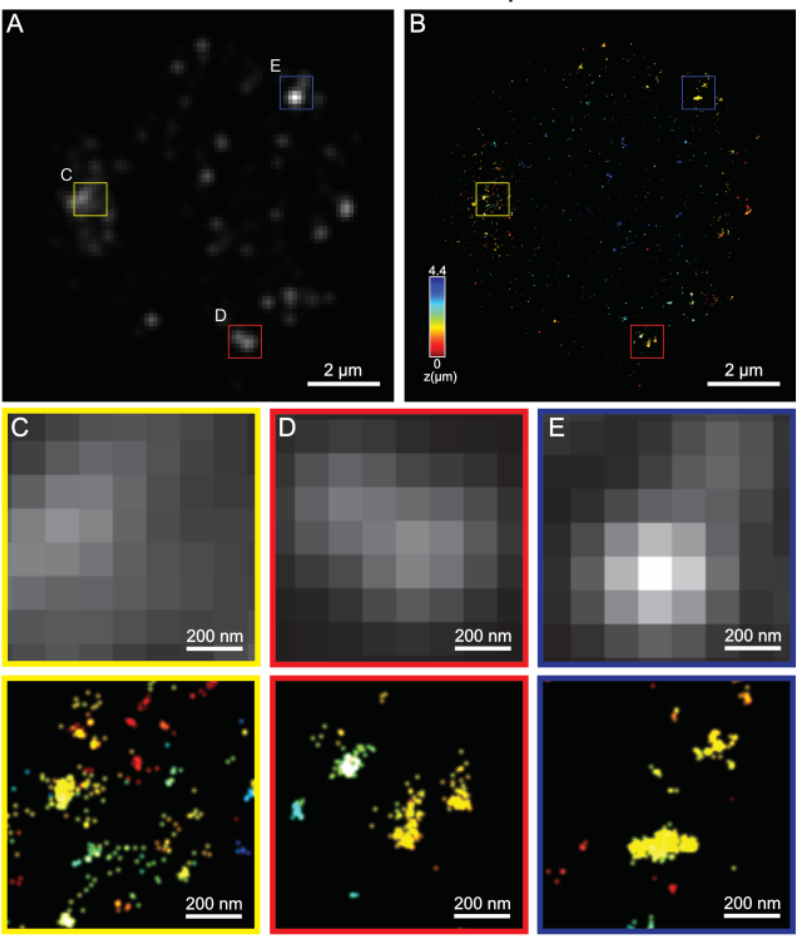

Figure 4. Single-molecule switching nanoscopy (SMSN) of cargo molecule on Jurkat T cell. (A) The diffraction-limited image of Alexa Fluor 647 labeled cargo molecules on the cell. Three boxes with different colors demonstrate cropped regions displayed in C-E. (B) 3D super-resolution image of A and each dot represents one emission event from a single molecule. The axial position of a single molecule is encoded in color, and the color bar indicating a $4.4 \mu \mathrm{m}$ axial range from the top (blue) to the middle part of the cell (red). (C-E) The enlarged cropped region from $A$ and their corresponding superresolution sub-regions from $B$.

recovered cargo to test for reusability (Figure S15). To estimate the extent of reusability, we performed the surface conjugation-disruption-reconjugation several times. We observed that our cargo can be reused at least two times in a reversible manner with the magnetically isolated reagent unlike a one-time use of existing reagents for live-cell imaging. To see whether the covalent cargo 2 can facilitate cell-cell interaction, we treated Jurkat $\mathrm{T}$ cells with this cargo for 30 minutes and then acquired bright-field 


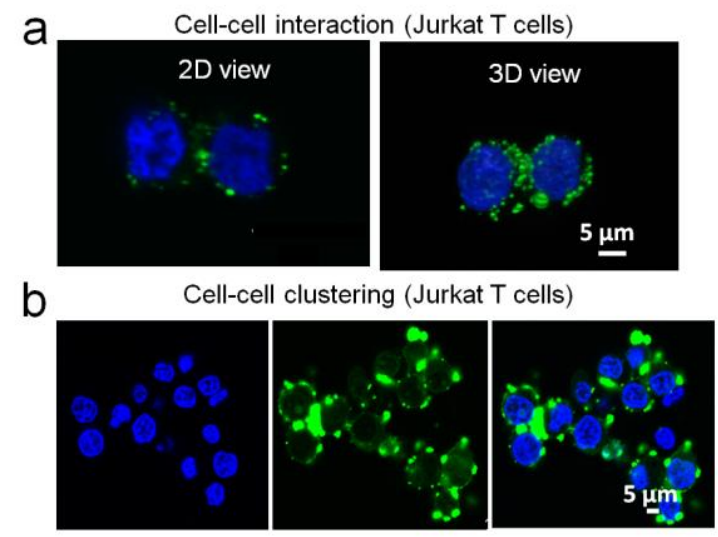

C

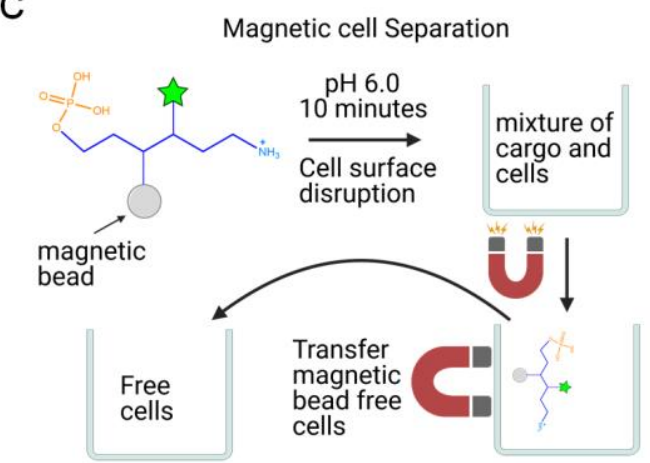

d

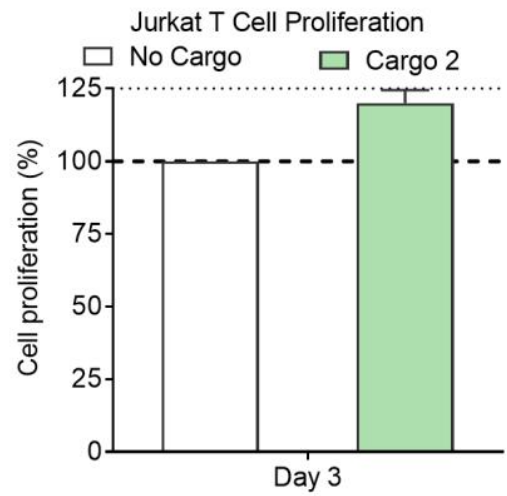

Figure 5. The effect of cell-cell interaction on the proliferation of T cells. (a) 2D and 3D confocal images of conjugated Jurkat $\mathrm{T}$ cells with the cargo molecule 2 . Scale bar $5 \mu \mathrm{m}$. (b) Confocal images of cell-cell clustering of Jurkat $T$ cells in presence of cargo molecule 2. Scale bar $5 \mu \mathrm{m}$. (c) Schematic. representations showing magnetic cell separation from a mixture of cargo and free cells. (d) Proliferation of Jurkat T cells in presence of the cargo 2 . Results are expressed as mean \pm SD. ${ }^{*} \mathrm{p}<0.05$ a statistically significant difference compared with the no cargo control group.

images of both the un-treated and cargo treated Jurkat $\mathrm{T}$ cells. This data revealed that untreated Jurkat $\mathrm{T}$ cells predominately exist as isolated cells in culture whereas cargo 2 treated Jurkat $\mathrm{T}$ cells formed clusters, indicating - cellcell interactions (Figure S16). Fluorescence images of the Jurkat $\mathrm{T}$ cells also revealed both cell-cell conjugation (Figure 5a) and cell-cell clustering (Figure 5b) phenomena for the cargo 2 treatment. To verify our cell-cell interactionbased proliferation hypothesis, we treated $10 \mu \mathrm{g} / \mathrm{mL}$ of the covalent cargo 2 with Jurkat T cells and performed a 3 days proliferation assay. We compared the results with cell culture growth media as vehicle control and the percentage of live cells in a cargo-treated sample was calculated relative to the vehicle control. We observed a statistically significant increase of $\sim 20 \%$ in the number of Jurkat $\mathrm{T}$ cells tread with cargo 2. To use our dual conjugation chemical strategy for enhancing the manufacturing of cell therapies, we functionalized magnetic beads to the cargo, since magnetic cargo molecule provides a simple method to separate cells from that cargo molecule (Figure 5c-d). This process can be repeated several times for faster proliferation of therapeutic cells and to retrieve the original cell culture every time (Figure S15). Thus, our cell conjugation method can be used reversibly to induce cell-cell communication and it is a much easier approach as compared to the other recently developed time-consuming and expensive methods such as DNA-programmed assembly of cells (DPAC). ${ }^{22,53,54}$

Finally, we were interested to study the change in proliferation and clustering of $T$ cells with time. We recorded realtime videos of conjugated and unconjugated Jurkat $\mathrm{T}$ cells by the IncuCyte live-cell analysis system that suggests an increase in clustering of surface conjugated $\mathrm{T}$ cells compared to vehicle treatment or no cargo control (Figure S17). Thus, our data clearly suggest the cargo reagent can reversibly functionalize cell membranes and can enhance cell-cell interactions which lead to an increase in the proliferation of therapeutic cells for bio-manufacturing.

\section{CONCLUSIONS}

We have developed a general, stable, and cytocompatible method of chemical conjugation on the live cell membranes and confirmed the conjugation by super-resolution imaging. One side chain of our cargo molecule is a positively charged ammonium moiety, which provides electrostatic binding resulting in non-covalent conjugation with the cell surface; while the other side contains a phosphate group bearing ADP moiety that forms phospho-ester covalent conjugation with the cell surface phosphates. This dual conjugated modification strategy resulted in long-time stability with the cell membrane and was non-toxic to live cells. While such an approach of chemical conjugation has many applications, we have shown an application of this method for live cell membrane imaging with long-time stability, recovery of the reagent after imaging, and reusability of the reagent for membrane imaging. Also, we have exploited this method for modulating cell-cell interaction leading to an increase in T cells in culture. Our conjugation method can be directly used to modify the live-cell surface functionalities without using additional cell-surface docking steps allowing for an easy-to-use reagent that can be simply adopted in any protocol. Also, no toxic effect was observed as revealed in the metabolic activity of the surface conjugated $\mathrm{T}$ cells. Therefore, our simple but powerful cell-functionalization strategy could be useful for cellbased therapeutics with wide-ranging applications. Future work of this technology includes enhancing the function of primary cells for future therapeutic applications. Given the general conjugation chemistry exploited by our method, we expect several other applications of this dualconjugation chemistry to functionalize membrane viruses, 
organisms (e.g. bacteria, yeast), etc. by conjugating with phospholipid moieties.

\section{ASSOCIATED CONTENT}

\section{Supporting Information.}

Copies of NMR and FT-IR spectra of the cargo, the procedure for the synthesis of the cargo, the procedure of superresolution imaging, procedure for cell surface conjugation are included in this document. This material is available free of charge via the Internet at http://pubs.acs.org.

\section{AUTHOR INFORMATION}

Corresponding Author

Gaurav Chopra - Department of Chemistry, Purdue University, 560 Oval Drive, West Lafayette, IN 47907, USA; Purdue Center for Cancer Research, Purdue University, 201 S University St, West Lafayette, IN 47906, USA; Purdue Institute for Integrative Neuroscience, Purdue University, 207 South Martin Jischke Drive, West Lafayette, IN 47907, USA; Purdue Institute for Inflammation, Immunology and Infectious Disease, Purdue University, 610 Purdue Mall, West Lafayette, IN 47907, USA; Purdue Institute for Drug Discovery, Purdue University, 720 Clinic Drive, West Lafayette, IN 47907, USA. orcid.org/00000003-0942-7898; Phone: 765-494-9264; Email: gchopra@purdue.edu

\section{Authors}

Ahad Hossain - Department of Chemistry, Purdue University, 560 Oval Drive, West Lafayette, IN 47907, USA

Joydeb Majumder - Department of Chemistry, Purdue University, 560 Oval Drive, West Lafayette, IN 47907, USA†

Cheng Bi - Purdue University, Weldon School of Biomedical Engineering, 206 S. Martin Jischke Drive, West Lafayette, IN 47907-2032

Fang Huang - Purdue University, Weldon School of Biomedical Engineering, 206 S. Martin Jischke Drive, West Lafayette, IN 47907-2032; Purdue Center for Cancer Research, Purdue University, 201 S University St, West Lafayette, IN 47906, USA; Purdue Institute for Integrative Neuroscience, Purdue University, 207 South Martin Jischke Drive, West Lafayette, IN 47907, USA; Purdue Institute for Inflammation, Immunology and Infectious Disease, Purdue University, 610 Purdue Mall, West Lafayette, IN 47907, USA.

\section{Author Contributions}

The manuscript was written through contributions of all authors. / All authors have given approval to the final version of the manuscript. / \$These authors contributed equally. (match statement to author names with a symbol)

\section{Funding Sources}

Any funds used to support the research of the manuscript should be placed here (per journal style).

Notes

There are no conflicts to declare.

\section{ACKNOWLEDGMENT}

This work was supported, in part, by the Department of Chemistry at Purdue University, Ralph W. and Grace M. Showalter Research Trust award, the Jim and Diann Robbers Grant for New Investigators Award, and NIH NCATS ASPIRE
Design Challenge awards to G.C. Additional support was, in part by, the Indiana Clinical and Translational Sciences Institute grant \# UL1TR002529 from NCATS, and the Purdue University Center for Cancer Research funded by the NIH grant \# P30 CA023168 is also acknowledged. F.H. was supported by the NIH (R35 GM119785) and DARPA (D16AP00093). The facilities at the Purdue Institute for Drug Discovery and Purdue Discovery Park were essential to conduct characterization and imaging experiments. The author(s) gratefully acknowledge the use of the facilities of the Bindley Bioscience Center, a core facility of the NIH-funded Indiana Clinical and Translational Sciences Institute. We thank the Purdue Imaging Facility for assistance with data collection. Figure 1 and $5 c$ were created with BioRender.com. The content is solely the responsibility of the authors and does not necessarily represent the official views of the National Institutes of Health.

\section{REFERENCES}

(1) Park, J.; Andrade, B.; Seo, Y.; Kim, M. J.; Zimmerman, S. C.; Kong, H. Engineering the Surface of Therapeutic "Living" Cells. Chem. Rev. 2018, 118 (4), 1664-1690. https://doi.org/10.1021/acs.chemrev.7b00157.

(2) Kellam, B.; De Bank, P. A.; Shakesheff, K. M. Chemical Modification of Mammalian Cell Surfaces. Chem. Soc. Rev. 2003, 32 (6), 327-337. https://doi.org/10.1039/b211643j.

(3) Li, M. D.; Atkins, H.; Bubela, T. The Global Landscape of Stem Cell Clinical Trials. Regen. Med. 2014, 9 (1), 27-39. https://doi.org/10.2217/rme.13.80.

(4) Gammon, J. M.; Dold, N. M.; Jewell, C. M. Improving the Clinical Impact of Biomaterials in Cancer Immunotherapy. Oncotarget 2016, 7 (13), 15421-15443. https://doi.org/10.18632/oncotarget.7304.

(5) Rabuka, D.; Forstner, M. B.; Groves, J. T.; Bertozzi, C. R. Noncovalent Cell Surface Engineering: Incorporation of Bioactive Synthetic Glycopolymers into Cellular Membranes. J. Am. Chem. Soc. 2008, 130 (18), 5947-5953. https://doi.org/10.1021/ja710644g.

(6) Vabbilisetty, P.; Boron, M.; Nie, H.; Ozhegov, E.; Sun, X.-L. Chemical Reactive Anchoring Lipids with Different Performance for Cell Surface Re-Engineering Application. ACS Omega 2018, 3 (2), 1589-1599. https://doi.org/10.1021/acsomega.7b01886.

(7) Borisenko, G. G.; Zaitseva, M. A.; Chuvilin, A. N.; Pozmogova, G. E. DNA Modification of Live Cell Surface. Nucleic Acids Res. 2009, 37 (4), 1-11. https://doi.org/10.1093/nar/gkn1034.

(8) Wang, T.; Leventis, R.; Silvius, J. R. Artificially Lipid-Anchored Proteins Can Elicit Clustering-Induced Intracellular Signaling Events in Jurkat T-Lymphocytes Independent of Lipid Raft Association. J. Biol. Chem. 2005, 280 (24), 22839-22846. https://doi.org/10.1074/jbc.M502920200.

(9) Custódio, C. A.; Mano, J. F. Cell Surface Engineering to Control Cellular Interactions. ChemNanoMat 2016, 2 (5), 376-384. https://doi.org/10.1002/cnma.201600047.

(10) Sakai, S.; Taya, M. On-Cell Surface Cross-Linking of Polymer Molecules by Horseradish Peroxidase Anchored to Cell Membrane for Individual Cell Encapsulation in Hydrogel Sheath. ACS Macro Lett. 2014, 3 (10), 972-975. https://doi.org/10.1021/mz5004322.

(11) Paulick, M. G.; Bertozzi, C. R. The Glycosylphosphatidylinositol Anchor: A Complex MembraneAnchoring Structure for Proteins. Biochemistry 2008, 47 (27), 6991-7000. https://doi.org/10.1021/bi8006324.

(12) Jia, H.-R.; Wang, H.-Y.; Yu, Z.-W.; Chen, Z.; Wu, F.-G. LongTime Plasma Membrane Imaging Based on a Two-Step Synergistic Cell Surface Modification Strategy. Bioconjug. Chem. 2016, $27 \quad$ (3), 782-789. https://doi.org/10.1021/acs.bioconjchem.6b00003.

(13) Kim, J. C.; Tae, G. The Modulation of Biodistribution of Stem Cells by Anchoring Lipid-Conjugated Heparin on the Cell Surface. J. Control. Release 2015, 217, 128-137. https://doi.org/10.1016/j.jconrel.2015.08.053. 
Lu, L.; Gao, J.; Guo, Z. Labeling Cell Surface GPIs and GPIAnchored Proteins through Metabolic Engineering with Artificial Inositol Derivatives. Angew. Chemie - Int. Ed. 2015, $54 \quad$ (33), 9679-9682. https://doi.org/10.1002/anie.201503814.

(15) Jewett, J. C.; Bertozzi, C. R. Cu-Free Click Cycloaddition Reactions in Chemical Biology. Chem. Soc. Rev. 2010, 39 (4), 1272-1279.

(16) Madl, C. M.; Heilshorn, S. C. Bioorthogonal Strategies for Engineering Extracellular Matrices. Adv. Funct. Mater. 2018 $28 \quad$ (11), 1706046 https://doi.org/10.1002/adfm.201706046.

(17) Wu, P.; Shui, W.; Carlson, B. L.; Hu, N.; Rabuka, D.; Lee, J.; Bertozzi, C. R. Site-Specific Chemical Modification of Recombinant Proteins Produced in Mammalian Cells by Using the Genetically Encoded Aldehyde Tag. Proc. Natl. Acad. Sci. 2009, $106 \quad$ (9), 3000-3005. https://doi.org/10.1073/pnas.0807820106.

(18) Stabler, C. L.; Sun, X.-L.; Cui, W.; Wilson, J. T.; Haller, C. A. Chaikof, E. L. Surface Re-Engineering of Pancreatic Islets with Recombinant Azido-Thrombomodulin. Bioconjug. Chem. 2007, 18 (6), 1713-1715. https://doi.org/10.1021/bc7002814

(19) Jennifer A. Prescher, D. H. D. \& C. R. B. Chemical Remodelling of Cell Surfaces in Living Animals. Nature 2004, 430 (7002), 870-873.

https://doi.org/https://doi.org/10.1038/nature02791.

(20) Krishnamurthy, V. R.; Wilson, J. T.; Cui, W.; Song, X.; Lasanajak, Y.; Cummings, R. D.; Chaikof, E. L. Chemoselective Immobilization of Peptides on Abiotic and Cell Surfaces at Controlled Densities. Langmuir 2010, 26 (11), 7675-7678. https://doi.org/10.1021/la101192v.

(21) Koo, H.; Lee, S.; Na, J. H.; Kim, S. H.; Hahn, S. K.; Choi, K. Kwon, I. C.; Jeong, S. Y.; Kim, K. Bioorthogonal Copper-Free Click Chemistry InVivo for Tumor-Targeted Delivery of Nanoparticles. Angew. Chemie - Int. Ed. 2012, 51 (47), 11836-11840. https://doi.org/10.1002/anie.201206703.

(22) Dutta, D.; Pulsipher, A.; Luo, W.; Yousaf, M. N. Synthetic Chemoselective Rewiring of Cell Surfaces: Generation of Three-Dimensional Tissue Structures. J. Am. Chem. Soc. 2011, 133 (22), 8704-8713. https://doi.org/10.1021/ja2022569.

(23) Pulsipher, A.; Dutta, D.; Luo, W.; Yousaf, M. N. Cell-Surface Engineering by a Conjugation-and-Release Approach Based on the Formation and Cleavage of Oxime Linkages upon Mild Electrochemical Oxidation and Reduction. Angew. Chemie Int. Ed. 2014, 53 (36), 9487-9492. https://doi.org/10.1002/anie.201404099.

(24) Pulsipher, A.; Griffin, M. E.; Stone, S. E.; Brown, J. M.; HsiehWilson, L. C. Directing Neuronal Signaling through CellSurface Glycan Engineering. J. Am. Chem. Soc. 2014, 136 (19), 6794-6797. https://doi.org/10.1021/ja5005174.

(25) Kim, H.; Shin, K.; Park, O. K.; Choi, D.; Kim, H. D.; Baik, S.; Lee, S. H.; Kwon, S. H.; Yarema, K. J.; Hong, J.; et al. General and Facile Coating of Single Cells via Mild Reduction. J. Am. Chem. Soc. 2018, $140 \quad$ (4), $1199-1202$ https://doi.org/10.1021/jacs.7b08440.

(26) Panza, J. L.; Wagner, W. R.; Rilo, H. L.; Rao, R. H.; Beckman, E. J.; Russell, A. J. Treatment of Rat Pancreatic Islets with Reactive PEG. Biomaterials 2000, 21 (11), 1155-1164.

(27) Inui, O.; Teramura, Y.; Iwata, H. Retention Dynamics of Amphiphilic Polymers PEG-Lipids and PVA-Alkyl on the Cell Surface. ACS Appl. Mater. Interfaces 2010, 2 (5), 1514-1520. https://doi.org/10.1021/am100134v.

(28) Jia, H. R.; Wang, H. Y.; Yu, Z. W.; Chen, Z.; Wu, F. G. Long-Time Plasma Membrane Imaging Based on a Two-Step Synergistic Cell Surface Modification Strategy. Bioconjug. Chem. 2016, $27 \quad$ (3), 782-789. https://doi.org/10.1021/acs.bioconjchem.6b00003.

(29) Tokunaga, T.; Kuwahata, K.; Sando, S. Systematic Exploration of Lipophilic Tags That Allow Efficient Anchoring of Aptamers to Live Cell Surfaces. Chem. Lett. 2013, 42 (2), 127-129. https://doi.org/10.1246/cl.2013.127.

(30) Matsuda, M.; Hatanaka, W.; Takeo, M.; Kim, C. W.; Niidome
T.; Yamamoto, T.; Kishimura, A.; Mori, T.; Katayama, Y. Short Peptide Motifs for Long-Lasting Anchoring to the Cell Surface. Bioconjug. Chem. 2014, 25 (12), 2134-2143. https://doi.org/10.1021/bc500465j.

Link, A. J.; Tirrell, D. A. Cell Surface Labeling of Escherichia c Oli via Copper(I)-Catalyzed [3+2] Cycloaddition. J. Am. Chem. Soc. 2003, 125 (37), 11164-11165. https://doi.org/10.1021/ja036765z.

(32) Riehemann, K.; Schneider, S. W.; Luger, T. A.; Godin, B. Ferrari, M.; Fuchs, H. Nanomedicine-Challenge and Perspectives. Angew. Chemie Int. Ed. 2009, 48 (5), 872-897. https://doi.org/10.1002/anie.200802585.

(33) Sanhai, W. R.; Sakamoto, J. H.; Canady, R.; Ferrari, M. Seven Challenges for Nanomedicine. Nat. Nanotechnol. 2008, 3 (5), 242-244. https://doi.org/10.1038/nnano.2008.114.

(34) Davila, M. L.; Riviere, I.; Wang, X.; Bartido, S.; Park, J.; Curran, K.; Chung, S. S.; Stefanski, J.; Borquez-Ojeda, O.; Olszewska, M.; et al. Efficacy and Toxicity Management of 19-28z CAR T Cell Therapy in B Cell Acute Lymphoblastic Leukemia. Sci. Transl. Med. 2014, 6 (224) https://doi.org/10.1126/scitranslmed.3008226.

(35) Kebriaei, P.; Poon, L. M. The Role of Allogeneic Hematopoietic Stem Cell Transplantation in the Therapy of Patients with Acute Lymphoblastic Leukemia. Curr. Hematol. Malig. Rep. 2012, 7 (2), 144-152. https://doi.org/10.1007/s11899-012-0116-3.

(36) Cheng, H.; Byrska-Bishop, M.; Zhang, C. T.; Kastrup, C. J.; Hwang, N. S.; Tai, A. K.; Lee, W. W.; Xu, X.; Nahrendorf, M.; Langer, R.; et al. Stem Cell Membrane Engineering for Cell Rolling Using Peptide Conjugation and Tuning of CellSelectin Interaction Kinetics. Biomaterials 2012, 33 (20), 5004-5012.

https://doi.org/10.1016/j.biomaterials.2012.03.065.

(37) Stephan, M. T.; Irvine, D. J. Enhancing Cell Therapies from the Outside in: Cell Surface Engineering Using Synthetic Nanomaterials. Nano Today 2011, 6 (3), 309-325. https://doi.org/10.1016/j.nantod.2011.04.001.

(38) Guinto, G.; Zepeda, E.; Londoño, D. Cell Therapy: Engineering and Manufacture of Pluripotent Cells. World Neurosurg. 2015, $\quad 84 \quad$ (5), $1209-1210$. https://doi.org/10.1016/j.wneu.2015.06.057.

(39) Tran, Khoi Q.; Zhou, Juhua; Durflinger, Katherine $H_{\text {.; }}$ Langhan, Michelle M.; Shelton, Thomas E.; Wunderlich, John R.; Robbins, Paul F.; Rosenberg, Steven A.; Dudley, M. E. Minimally Cultured Tumor-Infiltrating Lymphocytes Display Optimal Characteristics for Adoptive Cell Therapy. J. Immunother. 2008, $31 \quad$ (8), $\quad 742-751$. https://doi.org/10.1097/CJI.0b013e31818403d5.

(40) Kalos, M.; June, C. H. Adoptive T Cell Transfer for Cancer Immunotherapy in the Era of Synthetic Biology. Immunity 2013, 39 (1), 49-60. https://doi.org/10.1016/j.immuni.2013.07.002

(41) Wang, B.; Song, J.; Yuan, H.; Nie, C.; Lv, F.; Liu, L.; Wang, S. Multicellular Assembly and Light-Regulation of Cell-Cel Communication by Conjugated Polymer Materials. Adv. Mater. 2014, $26 \quad$ (15), 2371-2375 https://doi.org/10.1002/adma.201304593.

(42) Xiong, X.; Liu, H.; Zhao, Z.; Altman, M. B.; Lopez-Colon, D.; Yang, C. J.; Chang, L. J.; Liu, C.; Tan, W. DNA AptamerMediated Cell Targeting. Angew. Chemie - Int. Ed. 2013, 52 (5), 1472-1476. https://doi.org/10.1002/anie.201207063.

(43) Chapanian, R.; Constantinescu, I.; Brooks, D. E.; Scott, M. D. Kizhakkedathu, J. N. Invivo Circulation, Clearance, and Biodistribution of Polyglycerol Grafted Functional Red Blood Cells. Biomaterials 2012, 33 (10), 3047-3057. https://doi.org/10.1016/j.biomaterials.2011.12.053

(44) Griffin, M. E.; Hsieh-Wilson, L. C. Glycan Engineering for Cell and Developmental Biology. Cell Chem. Biol. 2016, 23 (1), 108-121. https://doi.org/10.1016/j.chembiol.2015.12.007.

(45) D’Souza, S.; Murata, H.; Jose, M. V.; Askarova, S.; Yantsen, Y.; Andersen, J. D.; Edington, C. D. J.; Clafshenkel, W. P.; Koepsel, R. R.; Russell, A. J. Engineering of Cell Membranes with a Bisphosphonate-Containing Polymer Using ATRP Synthesis for Bone Targeting. Biomaterials 2014, 35 (35), 9447-9458. 
Yun Lee, D.; Hee Nam, J.; Byun, Y. Functional and Histological

Evaluation of Transplanted Pancreatic Islets Immunoprotected by PEGylation and Cyclosporine for 1 Year. Biomaterials 2007, 28 (11), 1957-1966. https://doi.org/10.1016/j.biomaterials.2006.12.015.

(47) Prescher, J. A.; Bertozzi, C. R. Chemistry in Living Systems. Nat. Chem. Biol. 2005, 1 (1), 13-21. https://doi.org/10.1038/nchembio0605-13.

(48) Brannon-Peppas, L.; Blanchette, J. O. Nanoparticle and Targeted Systems for Cancer Therapy. Adv. Drug Deliv. Rev. 2004, 56 (11), 1649-1659. https://doi.org/10.1016/j.addr.2004.02.014.

(49) Davis, M. E.; Chen, Z.; Shin, D. M. Nanoparticle Therapeutics: An Emerging Treatment Modality for Cancer. Nat. Rev. Drug

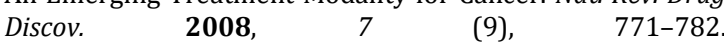
https://doi.org/10.1038/nrd2614.

(50) Gelderman, K. A.; Hultqvist, M.; Holmberg, J.; Olofsson, P.; Holmdahl, R. T Cell Surface Redox Levels Determine T Cell Reactivity and Arthritis Susceptibility. Proc. Natl. Acad. Sci. 2006, $\quad 103 \quad$ (34), 12831-12836. https://doi.org/10.1073/pnas.0604571103.

(51) Torres, A. G.; Gait, M. J. Exploiting Cell Surface Thiols to Enhance Cellular Uptake. Trends Biotechnol. 2012, 30 (4), 185-190. https://doi.org/10.1016/j.tibtech.2011.12.002.

(52) Gartner, Z. J.; Bertozzi, C. R. Programmed Assembly of 3 Dimensional Microtissues. Proc. Natl. Acad. Sci. 2009, 106 (17), 1-5.

(53) Todhunter, M. E.; Weber, R. J.; Farlow, J.; Jee, N. Y.; Cerchiari, A. E.; Gartner, Z. J. Fabrication of 3-D Reconstituted Organoid Arrays by DNA-Programmed Assembly of Cells (DPAC). Curr. Protoc. Chem. Biol. 2016, 8 (3), 147-178. https://doi.org/10.1002/cpch.8.

(54) Furst, A. L.; Klass, S. H.; Francis, M. B. DNA Hybridization to Control Cellular Interactions. Trends Biochem. Sci. 2019, 44 (4), 342-350. https://doi.org/10.1016/j.tibs.2018.10.002. 
For Table of Contents only

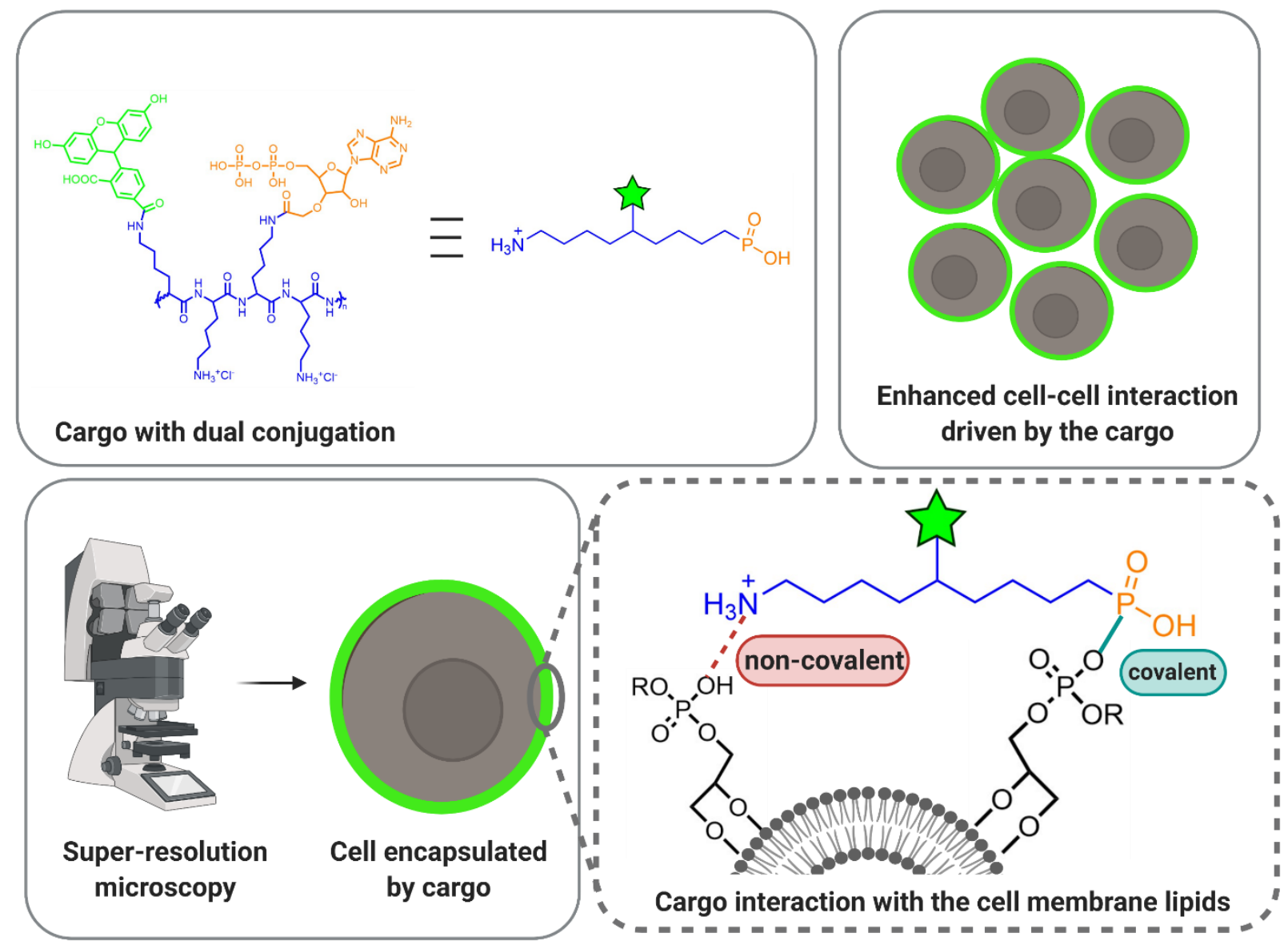

